\title{
Religion und Gesellschaft - Aktuelle Perspektiven
}

\author{
Matthias Koenig · Christof Wolf
}

Zusammenfassung: Mit dem Aufmerksamkeitsgewinn, den das Thema Religion in der Öffentlichkeit seit einigen Jahren erfährt, kann auch die Religionssoziologie einen neuen Aufschwung verzeichnen. Einleitend in das vorliegende religionssoziologische Sonderheft zeichnen wir die institutionelle und intellektuelle Entwicklung der deutschsprachigen Religionssoziologie seit der Nachkriegszeit nach und erläutern fünf aktuelle Trends des Faches. Sie betreffen Kontroversen um den Begriff der Religion, die Debatten um Säkularisierung, methodologische Fragen des soziologischen Erklärens, interdisziplinäre Forschung zu Religionen unter Bedingungen von Globalisierung sowie die Weiterentwicklung quantitativer und qualitativer Forschungsmethoden. Insgesamt deutet die Entwicklung in eine entschieden global-komparative Ausrichtung der Religionssoziologie, die wir mit einem knappen Ausblick auf zukünftige Forschungsperspektiven zusammenfassen.

Schlüsselwörter: Religion · Säkularisierung $\cdot$ Religionssoziologie

\section{Religion and society: current perspectives}

\begin{abstract}
Increasing public attention to religious questions has in the past years re-invigorated the sociology of religion. As an introduction to the present special issue we outline some institutional and intellectual developments of German language sociology of religion since the postwar period. We furthermore highlight five current trends of the subdiscipline that pertain to the concept of religion, to debates over secularization, to methodological questions of sociological explanation, to interdisciplinary research on religions under conditions of globalization, and to the refinement of quantitative as well as qualitative research methods. Having argued that these trends attest to an increasingly global-comparative approach, we conclude with some research perspectives for future sociology of religion.
\end{abstract}

Keywords: Religion $\cdot$ Secularization $\cdot$ Sociology of religion

(C) Springer Fachmedien Wiesbaden 2013

M. Koenig $(\bowtie)$

Institut für Soziologie, Georg-August-Universität Göttingen,

Platz der Göttinger Sieben 3, 37073 Göttingen, Deutschland

E-Mail: matthias.koenig@sowi.uni-goettingen.de

C. Wolf

GESIS Leibniz-Institut für Sozialwissenschaften,

Quadrat B 2, 68159 Mannheim, Deutschland

E-Mail: Christof.Wolf@gesis.org 


\section{Einleitung}

In der Soziologie galt das Schicksal der Religion in der Moderne lange als besiegelt. Zwar blieb der Versuch Auguste Comtes, die Soziologie als positive Wissenschaft an die Stelle der als überwunden geglaubten Religion zu setzen, in der Geschichte der Disziplin ohne größere Resonanz (vgl. Krech und Tyrell 1995). Aber dem sich im 19. Jahrhundert formierenden soziologischen Diskurs der Moderne war zumindest in Europa, anders als in den USA, wo die Soziologie zunächst eng mit der Social-Gospel-Bewegung verbunden war (vgl. Vidich und Lyman 1985), von Anfang an eine narrative Struktur der Säkularisierung eingeschrieben. Emile Durkheim, Georg Simmel und Max Weber sind nur einige unter Vielen, die bei aller Intensität des Nachdenkens über die historische Bedeutung der Religionen den christlichen Kirchen in arbeitsteiligen, individualisierten und rationalisierten Gesellschaften der Gegenwart einen dramatischen Bedeutungsverlust attestierten. Entsprechend wurde Religion in der zweiten Hälfte des 20. Jahrhunderts in einer zunehmend modernisierungstheoretisch ausgerichteten Soziologie als ein eher marginales Phänomen betrachtet.

Seit zwei Jahrzehnten etwa hat das Religionsthema indessen, wie markante Buchtitel dokumentieren (Graf 2004; Kepel 1991; Riesebrodt 2000), eine enorme Aufmerksamkeitskonjunktur erfahren, in der Soziologie wie überhaupt in den Geistes- und Sozialwissenschaften. Unübersehbar hatten in den 1970er Jahren die islamische Revolution, der christliche Fundamentalismus in den USA, der globale Erfolg der Pfingstbewegung von Lateinamerika bis Ostasien und neue Kontroversen um migrationsbedingte religiöse Pluralisierung die alte Gewissheit erschüttert, dass Religion für ein soziologisches Verständnis moderner Gesellschaften allenfalls von historischem Interesse sei. Theoretiker, die zunächst noch das säkulare Selbstbild der europäischen Moderne nachgezeichnet hatten, sahen sich zur grundlegenden Revision ihrer einstigen Annahmen gezwungen und sprachen nunmehr von „Desäkularisierung“ (Berger 1999) oder „,post-säkularen“ Gesellschaften (Habermas 2001).

Es liegt auf der Hand, dass die Religionssoziologie, lange selbst eine eher marginalisierte Teildisziplin, in dieser Situation einen neuen Aufschwung erfahren musste. Gerade die soziologische Forschung zu Veränderungen individueller Religiosität, zum Wandel der Sozialformen des Religiösen und zum Verhältnis der Religion zu anderen gesellschaftlichen Ordnungen verspricht schließlich Aufklärung über die Tragfähigkeit säkularer und post-säkularer Selbstbilder der Moderne. Es war daher durchaus an der Zeit, dass die Kölner Zeitschrift für Soziologie und Sozialpsychologie (KZfSS) nach zwanzig Jahren dem Religionsthema wieder ein eigenes Sonderheft widmete. Auslöser zur Idee eines neuen Sonderhefts war die erste Tagung des Arbeitskreises "Quantitative Religionsforschung“ im Jahr 2011 (vgl. Pollack et al. 2012), auf der dem Erstherausgeber deutlich wurde, dass es sich lohnen würde, aktuelle Perspektiven der Religionssoziologie in Deutschland und Europa auszuleuchten. Der Zweitherausgeber teilte diese Einschätzung aufgrund seiner Beschäftigung mit neueren Entwicklungen in der historisch-soziologischen Religionssoziologie. Beide nahmen daher den Auftrag des Herausgebergremiums der KZfSS, ein Sonderheft zum Thema Religion und Gesellschaft zu organisieren, ohne Zögern an.

Einleitend zu den hier versammelten Beiträgen zeichnen wir in einem ersten Schritt die Entwicklung der deutschsprachigen Religionssoziologie nach, um vor diesem Hin- 
tergrund die Schwerpunktsetzung des Sonderhefts zu begründen (Abschn. 2). In einem zweiten Schritt erläutern wir fünf aus unserer Sicht wichtige Trends der aktuellen Religionssoziologie, die sich auch in den einzelnen Beiträgen des vorliegenden Sonderhefts widerspiegeln (Abschn. 3). Wir schließen mit einem knappen Ausblick auf zukünftige Forschungsperspektiven der Religionssoziologie (Abschn. 4).

\section{Entstehung und Entwicklung des religionssoziologischen Feldes}

Bei allem Interesse, das dem Religionsthema in der Gründungsphase der Soziologie zukam, hat sich ein eigenes Feld der Religionssoziologie in den meisten europäischen Ländern erst seit dem Zweiten Weltkrieg formiert (vgl. für Frankreich Willaime 1999; für den angelsächsischem Raum Beckford 2000). Dies gilt auch für den deutschsprachigen Raum, auf den wir uns im Folgenden konzentrieren (vgl. Wohlrab-Sahr 2000). In institutioneller wie intellektueller Hinsicht unterscheiden wir holzschnittartig drei Phasen, die sich jeweils auch in den Inhalten der religionsbezogenen Sonderhefte der KZfSS widerspiegeln.

In der ersten Phase, der unmittelbaren Nachkriegszeit, waren es zunächst pastoralund kirchensoziologische Fragen, die das entstehende Feld der Religionssoziologie beherrschten (vgl. nur Goldschmidt et al. 1960). Dies hatte zumindest teilweise institutionelle Gründe. Waren es doch gerade die katholischen und protestantischen Kirchen, die in Reaktion auf den einsetzenden Schwund der eigenen Bindungskraft pastoralsoziologische Institute gründeten, empirische Sozialforschung finanzierten, Publikationsorgane schufen und damit nachhaltig die Infrastruktur für die Religionssoziologie prägten. Kirchenmitgliedschaft und Kirchgangshäufigkeit wurden seither zu Kernindikatoren empirischer religionssoziologischer Forschung. Bereits früh wurden innerhalb der Survey-Forschung auch differenzierte Messinstrumente für Spezialerhebungen zur Religiosität entwickelt. So enthielt die 1953 durchgeführte „Bundesstudie“ (vgl. Reigrotzki 1956) neben den beiden klassischen Indikatoren weitere Fragen zur Religiosität, wie beispielsweise die Teilnahme an kirchlichen Veranstaltungen, Fragen zu kirchlichen Nachrichten in der Zeitung und im Radio. Aber die überwiegende Zahl der Erhebungen, wie beispielsweise die 1972 erstmals durchgeführte Kirchenmitgliedschaftsuntersuchung der EKD, war eindeutig auf Kirchlichkeit als der nach wie vor vorherrschenden Gestalt des Religiösen in Deutschland konzentriert.

Ganz im Zeichen dieser kirchensoziologischen Fokussierung der Disziplin steht auch das älteste Sonderheft, das die KZfSS dem Religionsthema widmete (Goldschmidt und Matthes 1962). Dieses erste Heft gliederte sich in zwei Teile. Im ersten „Allgemeinen Teil“" griffen die Beiträge Fragen zur Ortsbestimmung der Religionssoziologie (Goldschmidt, Vrijhof, Mühlmann) und die damals noch lebendigen theoretischen Debatten um Ideologie- und Religionskritik auf (Birnbaum, Ludz). Interdisziplinäre Perspektiven wurden insbesondere durch theologisch orientierte Beiträge eingebracht (Mehl). Der „Spezielle Teil“ enthielt eine Vielzahl empirischer Studien, wobei auffällt, dass sich im Verhältnis zur Breite der Beiträge im ersten, allgemeinen Teil nahezu alle Untersuchungen auf die einsetzende Entkirchlichung sowie auf mit ihr verbundene kirchen- und pastoralsoziologische Fragen konzentrierten. Der regionale Fokus war keineswegs auf 
Deutschland beschränkt, sondern es wurden auch Entwicklungen in anderen Ländern Europas, Lateinamerikas und Asiens beleuchtet.

Dass der pastoral- und kirchensoziologische Zuschnitt der Religionssoziologie für deren Position innerhalb des soziologischen Feldes insgesamt nicht besonders förderlich war, liegt auf der Hand. Bereits früh fand daher die kirchensoziologische Verengung der Religionssoziologie in Thomas Luckmann (1960) und Joachim Matthes (1962) zwei prononcierte Kritiker. Indem sie eine Öffnung der Teildisziplin für die von den Klassikern gestellten großen Fragen nach der gesellschaftlichen Bedeutung von Religion und ihrem Schicksal in der Moderne forderten, legten sie wichtige Grundlagen für die spätere Entwicklung der Religionssoziologie.

Eine wichtige institutionelle Voraussetzung für die zweite Phase in der Entwicklung des religionssoziologischen Feldes waren die mit dem Hochschulausbau der 1970er Jahre einsetzenden Professionalisierungs- und Spezialisierungstendenzen innerhalb der Soziologie. Die damit verbundene Stärkung einer von kirchlich geförderter Pastoralsoziologie unabhängigen Religionssoziologie fand einen deutlichen Niederschlag in den deutschsprachigen Fachzeitschriften. Ein systematischer Durchgang durch die Jahrgänge der Kölner Zeitschrift für Soziologie und Sozialpsychologie, der Zeitschrift für Soziologie und der Sozialen Welt zeigt, dass gerade seit den 1980er Jahren vermehrt religionssoziologische Beiträge Eingang in den allgemeinen soziologischen Fachdiskurs fanden. Als Konsequenz dieser allmählichen Entwicklungen erfolgte nach ihrer Auflösung in den frühen 1970er Jahren im Jahr 1995 schließlich die Wiedergründung der DGS-Sektion Religionssoziologie, deren Agenda sich von pastoral- und kirchensoziologischen Fragen im engeren Sinn zunehmend löste.

Mit den veränderten institutionellen Rahmenbedingungen korrespondierten auch die intellektuellen Entwicklungen innerhalb des religionssoziologischen Feldes. In theoretischer Hinsicht ist zum einen das Bemühen um Anschluss an die Klassiker zu verzeichnen, das sich im starken Interesse an der werkgeschichtlichen und systematischen Rekonstruktion so wichtiger religionssoziologischer Theoretiker wie Max Weber (vgl. nur Schluchter 1988 sowie das beeindruckende Oeuvre von Hartmann Tyrell, z. B. 1993), Georg Simmel (Krech 1998), Norbert Elias (Hahn 1982, 1986) und auch Talcott Parsons (Brandt 1993) sowie in deren Anwendung auf historische und zeitgenössische Probleme (vgl. besonders Ebertz 1987, 1998) niederschlug. Zum anderen fanden zeitgenössische soziologische Großtheorien, insbesondere die Systemtheorie Niklas Luhmanns $(1972,1977)$ und Jürgen Habermas' Theorie des kommunikativen Handels, ein großes Echo in der Religionssoziologie (vgl. insgesamt Daiber und Luckmann 1983). In empirischer Hinsicht ist jene Phase vom Ausbau der nationalen, vor allem aber der internationalen Survey-Forschung geprägt. Neben den ALLBUS (zuerst 1980) traten nunmehr Survey-Instrumente wie die Europäische Wertestudie (zuerst 1981) oder das International Social Survey Programme (zuerst 1985), die aufgrund ihres Längsschnittcharakters auch die Beschreibung von Entwicklungstendenzen im religiösen Feld zuließen. Während diese Umfragen bis heute die klassischen Kernindikatoren von Kirchenmitgliedschaft und Kirchgangshäufigkeit enthalten, wird in dem stark durch Großbritannien geprägten European Social Survey (zuerst 2002), wie allgemein in angelsächsischen Ländern mangels eindeutiger Mitgliedschaftsregeln üblich, die „Neigung“ zu einer Kirche oder die individuelle Kirchenbindung erhoben (vgl. Wolf 2005). Zwar lag mit den Arbeiten von Charles Glock (1962) ein dezidiert mehrdi- 
mensionales Messinstrument vor, das religiösen Glauben, religiöses Verhalten, religiöse Erfahrung, religiöses Wissen und Konsequenzen der Religiosität auch jenseits von Kirchlichkeit differenziert zu untersuchen gestattete (vgl. ähnlich Boos-Nünning 1972). In die internationalen Surveys fand dieses Instrument jedoch allenfalls partiell Eingang.

Die genannten theoretischen und empirischen Entwicklungen basierten beide auf den damals in der Soziologie insgesamt vorherrschenden modernisierungstheoretischen Annahmen und gaben der Säkularisierungstheorie entsprechend einen beinahe paradigmatischen Status in der Religionssoziologie (vgl. Dobbelaere 1981; Tschannen 1992). Infolge der Rationalisierung kultureller Weltbilder, der Differenzierung gesellschaftlicher Ordnungen und der damit einhergehenden Individualisierung hätte institutionell verfasste Religion ihre einstige Bedeutung unwiederbringlich verloren. Eine ähnliche narrative Struktur liegt bei aller Kritik an der Säkularisierungstheorie selbst Luckmanns erst mit mehr als zwanzigjähriger Verspätung ins Deutsche übersetzten Theorie der unsichtbaren Religion (Luckmann 1991) zu Grunde, in deren Folge man sich zumal in der qualitativen Religionsforschung für individualisierte, populärkulturelle und massenmediale Erscheinungsformen des Religiösen interessierte, ohne dabei den modernisierungstheoretischen Rahmen insgesamt in Frage zu stellen.

Diese intellektuellen und institutionellen Entwicklungen einer neo-klassischen Religionssoziologie dokumentieren sich auch in dem von Bergmann, Hahn und Luckmann (1993) herausgegebenen zweiten religionsbezogenen Sonderheft der KZfSS. Fragen der Entkirchlichung im europäischen Kontext spielen hier weiterhin eine wichtige Rolle (Jagodzinski und Dobbelaere), deutlich ist aber auch der Einfluss der von Luckmann angestoßenen Debatte um Individualisierung und unsichtbare Religion erkennbar. So befassen sich etliche Studien mit nicht amtskirchlich verfassten Organisationsformen des Religiösen, etwa mit Sekten (Barker), fundamentalistischen Bewegungen (Leggewie) und New Age (Knoblauch) oder modernen Sinnanbietern (Hahn). Das Sonderheft stellt in regionaler Hinsicht eine deutliche Verengung gegenüber seinem Vorläufer dar, wird doch, von vereinzelten Beiträgen zu Frankreich, Italien oder der Schweiz abgesehen, der Blick selten über den deutschen Kontext hinaus gerichtet. Als besonders einflussreich erwiesen sich indessen einige Beiträge, die bereits eine grundsätzlichere Kritik an der Säkularisierungsthese und dem mit ihr verbundenen Religionsbegriff artikulierten. Matthes, der sich bereits im ersten Sonderheft kritisch zu einer vereinfachten Säkularisierungsthese geäußert hatte, wendet sich polemisch gegen die eurozentrische Verengung des Religionsbegriffs, und Tenbrucks historische Kultursoziologie des Religionsbegriffs schlägt in eine ähnliche Kerbe. Beide Beiträge weisen damit bereits voraus in die weitere Entwicklung des religionssoziologischen Diskurses.

Für die dritte Phase des religionssoziologischen Feldes sind zunächst wiederum veränderte institutionelle Rahmenbedingungen hervorzuheben. Insgesamt konnte das Fach auch in Deutschland von der Aufmerksamkeitskonjunktur profitieren, die der Religion aufgrund der eingangs genannten globalen Konstellation und insbesondere aufgrund der sichtbaren Präsenz des Islam im Westen seit den 1990er Jahren zuteil wurde und die sich seither in einer Reihe von Sonderforschungsbereichen, Graduiertenkollegs und Exzellenzclustern niedergeschlagen hat. Es haben sich in diesem Zusammenhang allerdings auch neue interdisziplinäre Konstellationen der Konkurrenz und Kooperation ergeben. Schon innerhalb der Soziologie interessiert man sich für Religion nicht mehr nur in der 
Religionssoziologie, sondern auch in der Allgemeinen Soziologie, der Kultursoziologie, der Organisationssoziologie, der Migrationssoziologie und der Geschlechterforschung, um einige der wichtigsten Teilbereiche zu nennen. Auch in Geschichtswissenschaft, Ethnologie, Politikwissenschaft und natürlich in der Religionswissenschaft befasst man sich, oftmals unter Rückgriff auf Methoden empirischer Sozialforschung, verstärkt mit religiösem Wandel in gegenwärtigen Gesellschaften. Und schließlich hat sich der religionssoziologische Diskurs, insbesondere in der Organisation empirischer Forschung, aber auch in Theoriedebatten, deutlich internationalisiert.

Die intellektuellen Trends dieser dritten Phase stellen wir detailliert im nächsten Abschnitt dar. An dieser Stelle genügt der Hinweis darauf, dass das modernisierungsund säkularisierungstheoretische Fundament des Faches nunmehr grundsätzlich in Frage gestellt und das Blickfeld deutlich über den europäischen oder westlichen Kontext hinaus erweitert wurde. Dieser veränderten Lage haben wir in der Konzeption des vorliegenden Sonderhefts des KZfSS Rechnung zu tragen versucht. So werden in einem ersten Abschnitt grundlegende handlungs-, organisations- und gesellschaftstheoretische Fragen des religiösen Wandels in modernen Gesellschaften erörtert. Es folgen, in konventioneller Gliederung nach eher mikro-, meso- und makrosoziologischen Problemen, drei Abschnitte, die sich im Einzelnen mit Entwicklungsdynamiken von individueller Religiosität, mit Sozialformen des Religiösen und schließlich mit dem Verhältnis von Religion zu anderen gesellschaftlichen Ordnungen befassen. Dabei wird der religiöse Wandel im deutschen und europäischen Kontext explizit mit nordamerikanischen und nicht-westlichen Situationen konfrontiert. Insgesamt soll mit dem Sonderheft ein bewusst breiter Querschnitt aktueller religionssoziologischer Forschung geboten werden, der gleichzeitig auch Diskussionen in den verwandten speziellen Soziologien und angrenzenden Disziplinen berücksichtigt.

\section{Aktuelle Trends der Religionssoziologie}

Blickt man nun genauer auf die dritte Phase der Religionssoziologie, so lassen sich fünf allgemeine intellektuelle Trends ausmachen, die sich auch in den Beiträgen dieses Sonderhefts niederschlagen. Sie betreffen die Kontroversen um den Religionsbegriff (3.1), die Debatten um Säkularisierung (3.2), die Frage nach allgemeinen Erklärungsmodellen innerhalb der Religionssoziologie (3.3.), die interdisziplinäre Erweiterung des Themenspektrums der Analyse von Religion unter Bedingungen von Globalisierung (3.4.) und die Methodenentwicklung in der sozialwissenschaftlichen Religionsforschung (3.5). Selbstverständlich ist mit diesen fünf Trends die Lage der Religionssoziologie nicht erschöpfend beschrieben. So bleibt gerade im deutschsprachigen Kontext die an die Klassiker anschließende Theorieentwicklung wichtig (vgl. z. B. Joas 2004). Wohl aber meinen wir, dass mit jenen Trends besonders wichtige intellektuelle Entwicklungen erfasst sind, die die internationale Religionssoziologie auch perspektivisch prägen werden.

\subsection{Kontroversen um den Religionsbegriff}

Wie wohl kaum eine andere Teildisziplin ist die Religionssoziologie seit jeher von grundlegenden Kontroversen über ihren genuinen Gegenstand geprägt. Eine erste, bereits auf 
die Klassiker zurückgehende Debatte, betrifft die Frage, ob Religion „substanziell“ über ihren symbolischen Gehalt oder „funktionell“ über ihre (individuelle oder gesellschaftliche) Funktion zu definieren sei (a). Eine zweite, neuere Diskussion betrifft die Frage nach der kulturellen Imprägnierung des (europäischen) Religionsbegriffs (b).

(a) Die Kontroverse zwischen „substanziellem“ und „funktionalem“ Religionsbegriff lässt sich bis zu den religionssoziologischen Theorien von Max Weber und Emile Durkheim zurückverfolgen. In der neo-klassischen Phase der Religionssoziologie wurden die beiden Definitionsstrategien von Peter Berger (1973) und Thomas Luckmann (1991) vertreten. Die Kontroverse zwischen Säkularisierungs- und Individualisierungstheoretikern (dazu näher unten) drehte sich nicht zuletzt um den jeweils zu Grunde gelegten engen oder weiten Religionsbegriff. Immer wieder hat es Versuche gegeben, beide Definitionsstrategien miteinander zu verbinden, man denke nur an die Bestimmung von religiöser Kommunikation als Kontingenzbewältigung im Modus der Codierung von Immanenz/ Transzendenz (vgl. prominent Luhmann 2000; Pollack 1995). Charakteristisch für die aktuelle Diskussionslage ist allerdings, dass sich aus den unterschiedlichen Definitionsstrategien letztlich eine Differenzierung verschiedener Forschungsstränge zu vollziehen scheint.

Als durchaus vital hat sich in den letzten Jahren die an Durkheim, Marcel Mauss und die Arbeiten des „Collège de Sociologie“ anschließende Soziologie erwiesen, die sich insbesondere für die mit dem Heiligen verbundenen, kreativen und destruktiven Kräfte interessiert (vgl. Möbius 2013). Sakralisierungen von Gewalt und Nationalismus wurden ebenso zum Thema wie die Sakralisierungen der natürlichen Umwelt oder der menschlichen Person (vgl. nur Joas 2011). Doch so fruchtbar diese Forschungstradition ist, es bleibt umstritten, was im Einzelnen damit gewonnen ist, den Religionsbegriff auf Phänomene auszudehnen, die ohne großen Verlust auch anders bezeichnet werden könnten (vgl. am Beispiel des Nationalismus Brubaker 2012). Nicht von ungefähr schlagen manche Vertreter dieser Forschungstradition daher inzwischen explizit vor, von einer Kultursoziologie des Sakralen zu sprechen (so etwa Lynch 2012).

Demgegenüber folgt man im Feld der Religionssoziologie vielfach der Tradition substanzieller Definitionsstrategien, wie immer die inhaltliche Bestimmung des Religionsbegriffs im Einzelnen vorgenommen wird (vgl. exemplarisch Riesebrodt 2007). Religion tritt damit von vornherein mit Weber als eigenlogische Wertsphäre oder als zumindest relativ autonomes soziales Feld (Bourdieu 2000) in den Blick. Das vorliegende Sonderheft konzentriert sich im Wesentlichen auf genau diese Forschungstradition. Selbst der Beitrag von Christoph Deutschmann, der in seiner Analyse des gegenwärtigen Kapitalismus als eines Werte und Visionen verkündenden und damit religioiden Systems entgrenzter Märkte einem funktionalen Religionsbegriff am nächsten kommt, folgt genau dort noch einem substanziellen Religionsbegriff, wo er den Modus der kapitalistischen Kontingenzbearbeitung von den Heilsversprechen der historischen Religionen unterscheidet.

(b) Gerade die substanzielle Definitionsstrategie ist indessen von Problemen betroffen, die in der zweiten hier anzusprechenden Kontroverse um den Religionsbegriff thematisiert wurden. Tenbruck und Matthes hatten bereits im oben genannten zweiten Sonderheft der KZfSS auf die kulturellen Voraussetzungen des europäischen Religionsbegriffs hingewiesen, der auch von der Soziologie unhinterfragt vorausgesetzt werde, und hatten 
angemahnt, Religion als „diskursiven Tatbestand“ (Matthes 1993) zu untersuchen. Zeitgleich hatte auch Talal Asad (1993), mit dem gesamten Instrumentarium Foucault'scher Diskursanalyse ausgestattet, den Blick etlicher Ethnologen, Historiker und schließlich auch Soziologen auf die Genealogien des Religionsdiskurses und dessen Machteffekte gelenkt. Tatsächlich hat sich in Folge der diskursanalytischen und begriffsgeschichtlichen Studien zum Religionsbegriff eine ganze Forschungsrichtung entwickelt, die sich mit der Pragmatik von Religionsdefinitionen befasst, also danach fragt, welche vor allem politischen und rechtlichen Voraussetzungen und Konsequenzen die diskursive Bezeichnung von Sachverhalten als „religiös“ oder „säkular“ hat (vgl. Platvoet und Molendijk 1999; Beyer 2006).

In diesem Sonderheft machen Monika Wohlrab-Sahr und Thomas Kaden symbolische Grenzziehungen zwischen Religion und Wissenschaft explizit zum Thema. Anhand eines Vergleichs der USA mit der DDR zeigen sie eindrücklich, dass diese Grenzziehung gesellschaftlich normiert ist, zum Gegenstand von Konflikten werden kann und prägend bis in die individuellen Haltungen und Identitäten von Nicht-Religiösen hineinwirkt. Auch Christel Gärtner trägt in ihrer Analyse der Religiosität Jugendlicher den alltagssprachlichen Religionssemantiken Rechnung, verbindet den an Matthes anschließenden diskursiven Religionsbegriff aber produktiv mit Ulrich Oevermanns Strukturmodell der Religiosität. Insgesamt, so wird man bilanzieren können, hat jene zweite Kontroverse um den Religionsbegriff dazu geführt, dass die historische und kulturelle Gebundenheit auch sozialwissenschaftlicher Religionsdiskurse deutlicher ins Bewusstsein gerückt ist.

\subsection{Die Diskussion um Säkularisierung}

Im Zentrum der inhaltlichen Diskussion innerhalb der Religionssoziologie steht, das kann angesichts der einleitenden Situationsbestimmung nicht weiter überraschen, nach wie vor die Säkularisierungstheorie (vgl. nur Gorski und Altinordu 2008). Bekanntlich war die Säkularisierungstheorie multidimensional angelegt, beinhaltete also Aussagen zu verschiedenen, zumindest analytisch voneinander zu trennenden Teilprozessen. Mit José Casanova (1994) lassen sich insbesondere der Rückgang religiösen Glaubens, die Privatisierung von Religion und die funktionale Differenzierung der Religion von anderen gesellschaftlichen Ordnungen als zentrale Dimensionen unterscheiden. Der Streit um die Säkularisierungstheorie betrifft inzwischen alle drei Dimensionen gleichermaßen.

Eine erste Runde des Streits bezog sich auf die These vom linearen Rückgang religiösen Glaubens, die im Wesentlichen aus zwei Richtungen kritisiert wurde. Einerseits wurde, inspiriert von Luckmanns Theorie unsichtbarer Religion, argumentiert, dass mit dem Übergang zur modernen Sozialstruktur nicht ein einfacher Rückgang, sondern vielmehr eine Individualisierung religiösen Glaubens zu beobachten sei, die sich in neuen Formen von Patchwork-Religiosität, Spiritualität und Ähnlichem niederschlage (vgl. exemplarisch Knoblauch 1989; für Frankreich Hervieu-Léger 2004; für England Heelas und Woodhead 2005). Andererseits wurde von Vertretern der in den USA entstandenen neueren Religionsökonomie argumentiert, dass der Rückgang von Kirchlichkeit in Europa nicht einem allgemeinen Modernisierungsprozess, sondern vielmehr der monopolitischen und regulierten Angebotsstruktur des dortigen religiösen Marktes geschuldet sei; gerade der Vergleich mit den USA zeige nämlich, dass Kirchlichkeit unter spezifi- 
schen Bedingungen, Deregulierung und Pluralisierung, in modernen Gesellschaften sogar zunehmen könne (vgl. exemplarisch Stark und Iannaconne 1994).

Die Debatte zwischen Vertretern der Säkularisierungstheorie, der Individualisierungsthese und des Marktmodells, die eine Fülle historischer und empirischer Detailbefunde $\mathrm{zu}$ Tage förderte, ist bis heute alles andere als abgeschlossen (vgl. umfassend Pollack 2009). Eine erste wichtige Detailfrage innerhalb dieser Debatte betrifft die Periodisierung. Während oftmals der Wertewandel der 1960er Jahre für eine (beschleunigte) Säkularisierung verantwortlich gemacht wird, zeigen Historiker, dass es bereits viel früher deutliche Entkirchlichungs- und Säkularisierungstendenzen, allerdings auch gegenläufige Prozesse gegeben hat (z. B. McLeod 2000). Auf Basis der kirchlichen Teilnahmestatistiken für Deutschland zwischen 1900 und 1960 wird diese Frage in diesem Sonderheft von Benjamin Ziemann untersucht. Er kommt zu dem Schluss, dass die konfessionellen Milieus bereits vor 1945 deutlich an Bindungskraft verloren haben. Neben der Periodisierungsfrage ist in der Debatte zweitens der Vergleich der europäischen mit der nordamerikanischen Entwicklung von strategischer Bedeutung. Gegenüber der Markttheorie sind hier inzwischen alternative Erklärungsmodelle formuliert worden; Norris und Inglehart (2004) beispielsweise betonen statt des (fehlenden) religiösen Marktes den Einfluss des Wohlfahrtsstaates, der viele existenzielle Unsicherheiten beseitigt oder zumindest abmildert und damit die Nachfrage nach Religion verringert. Andere betonen demgegenüber Unterschiede langfristig wirksamer Religionskulturen (Berger et al. 2008). Tatsächlich zeichnet sich, drittens, innerhalb der Debatte insgesamt ab, dass neben den genannten drei theoretischen Argumenten der moderierende Einfluss historisch geprägter Religionskulturen auf religiöse Einstellungen und Handlungsmuster in Rechnung zu stellen ist, und zwar nicht nur im transatlantischen, sondern auch im innereuropäischen Vergleich, wo ebenfalls höchst unterschiedlich verlaufende Entkirchlichungs- und Säkularisierungsprozesse zu konstatieren sind (vgl. Pickel 2010; Voas 2009). In diesem Sonderheft wird dieses Argument von Olaf Müller, Detlef Pollack und Gert Pickel entfaltet. Gestützt auf verschiedene quantitative Datenquellen zeigen sie im ost-/westdeutschen Vergleich, dass die jeweils vorherrschende konfessionelle Mehrheitskultur sogar die jeweilige Minderheitskultur beeinflusst. Sie argumentieren, dass die dominant konfessionelle Kultur im Westen die Entkirchlichungs- und Säkularisierungsprozesse eher bremst, während die säkular-konfessionslose Mehrheitskultur im Osten beschleunigend auf die Säkularisierung wirkt. In ihrer qualitativ angelegten Untersuchung der religiösen Identitäten von Jugendlichen aus Ost- und Westdeutschland kommt Gärtner zu ganz ähnlichen Befunden. Kulturelle Rahmungen gelten schließlich, viertens, auch in einer neuen Forschungsrichtung als relevant, die sich der inneren Sinnstruktur dezidiert nicht-religiöser oder säkularer Haltungen widmet (Wohlrab-Sahr et al. 2009; Zuckermann 2009). So entwickeln Wohlrab-Sahr und Kaden in ihrem bereits erwähnten Beitrag eine Typologie unterschiedlicher Modi von Nicht-Religiosität, die jeweils auf die umgebenden Kontexte bezogen werden.

Die zweite Runde des Streits um die Säkularisierungstheorie geht über die skizzierte Debatte insofern hinaus, als in ihr die These einer Privatisierung der Religion hinterfragt wurde. Casanovas (1994) breit rezipierte Studie zu öffentlicher Religion in katholisch geprägten Gesellschaften markierte den Auftakt zu dieser Debatte. Ihm zufolge ist es durchaus möglich (und in normativer Hinsicht legitim), dass religiöse Akteure auch unter 
Bedingungen der Moderne sozialmoralische und politische Fragen thematisieren und damit den öffentlichen Diskurs demokratischer Gesellschaften beeinflussen (vgl. neuerdings auch Hennig 2012).

Auch hier sind die Fragen alles andere als geklärt, wie die Beiträge in diesem Sonderheft dokumentieren. Auf der einen Seite kann Ateş Altinordu in seinem historischen Vergleich der Politisierung des Katholizismus im deutschen Kaiserreich mit dem politischen Islam in der kemalistischen Türkei nachweisen, dass und wie zunächst anti-modernistische religiöse Bewegungen zu wichtigen Akteuren der öffentlichen Sphäre und der politischen Gesellschaft werden. Auf der anderen Seite sind gerade im europäischen Kontext Auflösungserscheinungen solcher konfessioneller Milieus zu verzeichnen, die eine massenbasierte Politisierung erschweren. So zeigen Sigrid Roßteutscher und Christof Wolf in ihrem Beitrag, gestützt auf Datenreihen des deutschen ALLBUS, dass in Deutschland der Zusammenhang von Religiosität mit politischen Einstellungen und Verhaltensweisen im Zeitverlauf zurückgeht, was eher die säkularisierungstheoretische These der Privatisierung von Religion bestätigt.

Bei aller Kritik an der Säkularisierungstheorie, die in diesen Debatten um öffentliche Religion bereits geäußert wurde, blieb ihr differenzierungstheoretischer Kern lange weitgehend unangetastet. Genau hier setzt die dritte und jüngste Runde in der Kontroverse um die Säkularisierungstheorie an. In ihr wird mit dem Differenzierungskonzept letztlich der modernisierungstheoretische Kern des Säkularisierungsparadigmas zur Disposition gestellt (vgl. Casanova 2006). Stand die Modernisierungstheorie ohnehin bereits seit längerem in der Kritik, vor allem seitens einer kontingenzbewussten historischen Soziologie (Knöbl 2007), so werden entsprechende Revisionen nunmehr auch in der Religionssoziologie gefordert. Philip Gorski (2000, 2003) etwa kritisiert das Differenzierungstheorem insbesondere dafür, dass es die konfessionelle Staatlichkeit der frühen europäischen Neuzeit, aber auch spätere (zivil-)religiöse Codierungen von Nationen nur schwer zu fassen vermag. Ist die Vorstellung einer linearen Differenzierung von Religion und Politik damit schon für die europäische Entwicklung strittig, so ist seine Tragfähigkeit für die Beschreibung außereuropäischer Entwicklungen nur noch strittiger. Dies zeigt sich besonders in der von Shmuel N. Eisenstadt (2000) angestoßenen Debatte um „,multiple Modernitäten“, in der Variationen der Differenzierungs- und Integrationsmuster moderner Gesellschaften auf tieferliegende ,,achsenzeitliche“ Traditionen und deren Transformation zurückgeführt werden. In der Religionssoziologie hat diese Debatte, die durch Charles Taylors (2007) monumentale Analyse säkularer Kultur an Fahrt gewonnen hat, ein breites Echo gefunden und eine neue Forschungsliteratur zu ,multiplen Säkularitäten“ angeregt (Koenig 2011; Wohlrab-Sahr und Burchardt 2012), die auch in diesem Sonderheft mit dem Beitrag von Wohlrab-Sahr und Kaden repräsentiert ist.

So intensiv die Diskussion um die klassische Differenzierungstheorie inzwischen auch im deutschsprachigen Raum geführt wird (vgl. nur Gabriel et al. 2012; Willems et al. 2013), so offen bleibt bislang indessen, was an ihre Stelle treten soll. Ein Vorschlag wird in diesem Sonderheft von Thomas Schwinn unterbreitet. Er lotet die neuere religionssoziologische Diskussion auf ihre Implikationen für die Differenzierungstheorie aus und plädiert für ein an Weber orientiertes Forschungsprogramm, innerhalb dessen verschiedene Wege und Muster der Differenzierung religiöser, politischer und anderer Ordnungen konflikt- und spannungstheoretisch analysiert werden. 


\subsection{Säkularisierung, Religion und das Problem soziologischen Erklärens}

Die oben skizzierte Debatte um Säkularisierung kreiste in allen drei Runden um die Beschreibung langfristiger Entwicklungstrends und entsprechende Gegenwartsdiagnosen. Wie die Modernisierungstheorie war die Säkularisierungstheorie indessen einer noch grundlegenderen Kritik ausgesetzt, die das methodologische Problem der jeweils unterstellten Modelle zur Erklärung makrosoziologischer Prozesse betrifft. Zwei Kontroversen sind in diesem Zusammenhang zu erwähnen.

An erster Stelle ist die Kontroverse um die kausale Bedeutung von Religion zu nennen. Diese Kontroverse, die vor allem im angelsächsischen Bereich intensiv geführt wird, wurde von allgemeinen Entwicklungen in der Kultursoziologie angeregt, in deren Folge um die konstitutive Bedeutung von kollektiven Repräsentationen, Symbolen und Modellen gerungen wurde (vgl. allgemein Reckwitz 2001). Der von Jeffrey C. Alexander (2003) geprägte Begriff des ,strong program“ der Kultursoziologie ist auch für eine Religionssoziologie in Anspruch genommen worden, die der Religion, durchaus auch im substanziellen Sinne definiert, eine starke kausale Wirkung zuschreibt. Tatsächlich zeugt die bereits angesprochene Debatte um multiple Modernitäten, aber auch die historisch-soziologische Forschung zu konfessionellen Wurzeln des Wohlfahrtsstaats (Manow 2008) und Nationalismus (Spohn 2008) sowie auch die quantitative Forschung zu religiösen Determinanten von Geschlechtereinstellungen (Inglehart und Norris 2003) oder sozialem Kapital (Putnam und Campbell 2010), von der Fruchtbarkeit dieser Perspektive. Gleichwohl wurde von Autoren wie David Smilde jüngst auf Probleme einer allzu pauschalen Inanspruchnahme von Religion als explanatorischem Faktor hingewiesen und ein „weak program“ der Religionssoziologie eingefordert (vgl. seinen Beitrag in Bender et al. 2012). Wie in der Kultursoziologie allgemein, so ist auch in der Religionssoziologie eine Entscheidung zwischen diesen beiden gegenläufigen Erklärungsprogrammen, die in dem Beitrag von Deutschmann am Beispiel der Debatte um (religiöse) Ideen und (ökonomische) Interessen facettenreich ausgeleuchtet werden, indessen nicht a priori zu treffen. Es bleibt vielmehr, wie der Beitrag von Roßteutscher und Wolf in diesem Sonderheft dokumentiert, letztlich eine empirisch zu klärende Frage, welche kausale Bedeutung dem religiösen Faktor zukommt und wie sich dieser über die Zeit verändert.

Die zweite und grundlegendere Kontroverse ist von Entwicklungen innerhalb der Allgemeinen Soziologie angestoßen worden, die an jede soziologische Erklärung den Anspruch auf Rekonstruktion wirksamer kausaler Mechanismen stellt. Auf der einen Seite wird dieses Argument in der analytischen Soziologie vertreten, in der man makrosoziale Explananda generell als Aggregation der ihrerseits situativ bedingten Handlungsselektionen von Individuen zu erklären versucht (vgl. exemplarisch Hedström 2005). Auf der anderen Seite findet man es auch in der im angelsächsischen Raum lebendigen Tradition des kritischen Realismus (Gorski 2009; Smith 2010), die die von der analytischen Soziologen unterstellte Prämisse des methodologischen Individualismus für unnötig hält und demgegenüber von einer geschichteten sozialen Wirklichkeit ausgeht, in der kausale Mechanismen auf verschiedenen Ebenen wirksam sind.

Auch in der Religionssoziologie sind diese Debatten um die Logik soziologischen Erklärens inzwischen, wenn auch zögerlich, rezipiert worden. Unabhängig von der Kontroverse um die zutreffende Beschreibung religiösen Wandels wurde die Säkulari- 
sierungstheorie etwa dafür kritisiert, dass sie in allen drei Dimensionen die jeweils wirksamen kausalen Mechanismen unterspezifiziert lasse (vgl. beispielsweise Smith 2003). Schon die an der Handlungstheorie der rationalen Wahl orientierte Religionsökonomie unternahm wichtige, wenngleich einseitige Vorstöße, genau dieses Defizit auszugleichen (Stark 1999). In diesem Sonderheft ist es Jörg Stolz, der die Stoßrichtung der analytischen Soziologie religionssoziologisch fruchtbar $\mathrm{zu}$ machen versucht. Ausgehend von einer substanziellen Definition von Religion skizziert er ein Modell religiös-säkularer Konkurrenz, das eine Vielzahl von Einzelbefunden aus Entscheidungen zwischen religiösen und säkularen Gütern herleitet, die ihrerseits von Kontextfaktoren, wie der Regulierung von Angebot und Nachfrage und dem Modernisierungsgrad abhängen. Die an der Tradition des kritischen Realismus orientierte Konzeption sozialer Mechanismen hat in der Religionssoziologie bislang dagegen weniger Aufmerksamkeit erfahren. Eine Ausnahme stellen die grundlagentheoretischen und historisch-soziologischen Arbeiten von Gorski (2003) dar, der unter Rückgriff auf Einsichten von Weber, Foucault und Bourdieu relationale Mechanismen religiösen Wandels zu erfassen versucht. In diesem Sonderheft ist diese Richtung durch den Beitrag von Altinordu vertreten, der in seinem historisch-soziologischen Vergleich von politischem Katholizismus und politischem Islam generalisierbare Mechanismen zu rekonstruieren versucht, in denen religiöse Bewegungen politisiert werden, bei zunehmender Einbeziehung in den demokratischen Prozess indessen ihre zunächst modernitätsfeindlichen Positionen abmildern.

\subsection{Religionen in der gegenwärtigen Weltgesellschaft}

So zentral die Säkularisierungsdiskussion nach wie vor für die Religionssoziologie ist, hat sich an ihren Rändern eine Vielzahl neuer Forschungsfelder formiert, in denen die Eigendynamik von Religion unter Bedingungen von Globalisierung thematisiert und damit eine stärker komparative Ausrichtung der Teildisziplin sichtbar wird. Vier Forschungsfelder möchten wir im Folgenden selektiv in den Vordergrund rücken, weil sich in ihnen besonders deutlich der oben angesprochene intensivierte Dialog mit angrenzenden Teildisziplinen zeigt.

Ein erstes Forschungsfeld ist die zunehmend lebendige Literatur zur Religion im Prozess des globalen Migrationsgeschehens. Migration ist ein wichtiger Faktor religiöser Pluralisierung. Sie kann religiöse Identitäten revitalisieren und löst, wie insbesondere Debatten um den Islam in Europa zeigen (Tezcan 2003), oftmals religionspolitische Kontroversen aus. Umso wichtiger ist daher das Verständnis der Prozesse des Wandels religiöser Überzeugungen und Praktiken, die bei Migranten und ihren Nachkommen stattfinden. Die Migrationssoziologie bietet in diesem Zusammenhang verschiedene Erklärungsmodelle, angefangen von klassischen Assimilationstheorien bis hin zu Theorien reaktiver Ethnizität, die inzwischen auch für die Analyse von Religiosität fruchtbar gemacht werden (vgl. umfassend Breton 2012). Wie sich Migration auf die Religiosität von Migranten auswirkt, untersuchen in diesem Sonderheft Claudia Diehl und Matthias Koenig. Auf der Basis neuer Daten unter türkischen und polnischen Zuwanderern weisen sie nach, dass die religiöse Partizipation unmittelbar nach der Zuwanderung aufgrund mangelnder Opportunitätsstrukturen im Vergleich zur Partizipation im Herkunftsland sinkt, um dann aber zumindest bei türkischen Muslimen aufgrund symbolischer Grenzdynamiken 
im Zeitverlauf wieder anzusteigen. Die Bedeutung salienter symbolischer Grenzen für die Stabilisierung religiöser oder religiös-ethnischer Identitäten zeigt auch der Beitrag von Gärtner, die in ihrer Analyse der religiösen Identität Jugendlicher u.a. qualitative Befunde zu jungen Musliminnen präsentiert. Ein extremes Beispiel einer ehemals aus Migration entstandenen ethnisch-religiösen Gemeinschaft steht im Mittelpunkt des Beitrags von Dorothea Lüddeckens und Rafael Walthert. Sie untersuchen, wie Parsi Zoroastrier in Mumbai die Herausforderungen bewältigen, die für sie durch die Modernisierung Indiens und die damit verbundene Mobilität entstanden sind.

Die Analyse ethnisch-religiöser Vergemeinschaftung verweist gleichzeitig auf ein zweites äußerst lebendiges Forschungsfeld, das sich mit den Sozialformen des Religiösen befasst. Waren die diesbezüglichen Fragen in der Religionssoziologie lange unter Rückgriff auf die klassischen Unterscheidungen von anstaltsförmiger „Kirche“ und freiwilliger „Sekte“ thematisiert worden, so werden nunmehr verstärkt Einsichten der allgemeinen Organisationssoziologie, sei sie neo-institutionalistischer oder systemtheoretischer Provenienz, aufgegriffen (vgl. Gabriel et al. 1998; Hero 2010). In diesem Sonderheft wird die Vielfalt der Sozialformen von Religion in dem Beitrag von Volkhard Krech, Jens Schlamelcher und Markus Hero ausgeleuchtet. Ausgehend von der Instabilität religiöser Kommunikation (dazu näher Tyrell et al. 1998) unterscheiden sie idealtypisch die Sozialformen von Gemeinschaft, Organisation und Marktbeziehungen sowie, davon abgeleitet, soziale Bewegungen und Events. Sie argumentieren, dass es in modernen Gesellschaften, wie Entwicklungen sowohl innerhalb als auch außerhalb der christlichen Großkirchen zeigen, gerade die Kombination verschiedener Sozialformen ist, in der sich religiöse Kommunikation auch in modernen Gesellschaften stabilisieren lässt. Eine systemtheoretische Perspektive auf religiöse Organisationen wird in dem Beitrag von Thomas Kern und Uwe Schimank zu Mega-Churches in den USA eingenommen. Ihr Argument lautet, dass der Erfolg dieser in einer prononcierten Wachstumsdoktrin begründeten Kirchen daher rührt, dass sie Elemente freiwilliger Gemeinschaft in programmatischer, personeller und kommunikativer Hinsicht in eine ansonsten formalisierte Organisationsstruktur integrieren.

Die hier untersuchten Sozialformen von Religion verweisen auf ein drittes Forschungsfeld, das sich den globalen religiösen Bewegungen der Gegenwart widmet. Die Religionssoziologie steht hier in engem Dialog mit Forschungen in Ethnologie, Religions- und Regionalwissenschaften, die ihren Ausgang unter anderem in dem religionsvergleichend angelegten Forschungsprojekt zum Fundamentalismus im Christentum, Judentum und Islam genommen haben (Marty und Appleby 1991). Mittlerweile richtet sich das Interesse aber nicht alleine auf die legalistischen und charismatischen Fundamentalismen (Riesebrodt 1990) und auf deren Gewaltpotenzial (Kippenberg 2008). Vielmehr sind auch andere Bewegungen von den Missionsbewegungen innerhalb der kolonialen Imperien (van der Veer 2001) bis hin zum gegenwärtigen pfingstlerischen Christentum in den Blick gerückt (Martin 1990; Höllinger 2007). Während der bereits erwähnte Beitrag von Kern und Schimank spezifische organisatorische Strukturen einer auf Wachstum angelegten Religionsgemeinschaft beleuchtet, thematisiert Thomas Kirsch gestützt auf langjährige ethnographische Forschung in Pfingstgemeinden in Sambia einen bislang wenig beachteten Aspekt der pentekostalen Ausbreitungsdynamik, nämlich die in der Ontologie des 
Heiligen Geistes selbst angelegten sozialen Beziehungen, und macht auf lokale kulturelle Voraussetzungen für die Resonanz missionarischer Bewegungen aufmerksam.

Migrationsbedingte Pluralisierungsprozesse, der Wandel von Sozialformen des Religiösen und die globalen religiösen Bewegungen stellen weltweit neue Herausforderungen an staatliches Handeln. Entsprechend befasst sich ein viertes Forschungsfeld, das an der Schnittstelle von Interessen der Religionsökonomie für regulatorische Regime, von Debatten um multiple Säkularitäten und von neuen Strömungen der vergleichenden Politikwissenschaft entstanden ist, mit Dynamiken der Religionspolitik im internationalen Vergleich. Hat die Literatur zunächst Typologien von Staat-Kirche-Beziehungen oder allgemein von religionspolitischen Regimen entfaltet, so interessiert man sich zunehmend für die, sei es binnenstaatlichen, sei es weltgesellschaftlichen, kausalen Mechanismen, die Entstehung, Pfadabhängigkeit und institutionellen Wandel religionspolitischer Regime (vgl. etwa Koenig 2007; Gill 2008; Kuru 2009) sowie deren Konsequenzen für religiöse Konflikte zu erklären vermögen (Grim und Finke 2011). Im vorliegenden Sonderheft geht Christian Joppke in deutsch-französischer Vergleichsperspektive auf die Bedeutung der gerichtlichen Arena für die Integration des Islam ein. Er skizziert Unterschiede zwischen individualrechtlichen und korporativen Logiken der rechtlichen Berücksichtigung religiöser Minderheitenbelange und erläutert die Spannungen, die in deren Folge innerhalb demokratischer Meinungs- und Willensbildungsprozesse entstehen können.

\subsection{Methodenentwicklung in der Religionssoziologie}

Die bislang skizzierten theoretischen und thematischen Trends der Religionssoziologie werden dadurch flankiert, dass sich mit der Professionalisierung und Spezialisierung des Subfeldes auch die Methoden der Religionssoziologie deutlich weiterentwickelt haben.

Dies zeigt sich, erstens, in der quantitativen Religionsforschung. Nach wie vor sind Kirchenmitgliedschaft und Kirchgangshäufigkeit die geläufigsten Indikatoren von Religiosität. Für eine historische Prüfung der ersten Komponente der Säkularisierungstheorie ist es von großer Bedeutung, auch die Zeit vor dem Einsetzen umfangreicher SurveyForschung zu analysieren. Man sieht sich daher auf die kircheneigenen Statistiken zu Gottesdienstbesuch, Abendmahlsteilnahme, Taufen, Kirchenaustritten und ähnlichem verwiesen, deren Probleme in dem Beitrag von Ziemann kritisch diskutiert werden. Für eine Prüfung der Säkularisierungstheorie und ihrer Alternativen ist allerdings auch eine Verfeinerung der Messinstrumente von Religiosität erforderlich. Die oben erwähnten Arbeiten von Glock und Boos-Nünning stellten einen wichtigen Bezugspunkt dar, als die quantitative Religionsforschung in Deutschland einen neuen Aufschwung erfuhr. So unterscheiden Kecskes und Wolf $(1993,1995)$ in ihrem Messinstrument zur Erfassung christlicher Religiosität die Dimensionen christlicher Glaube, christliche Erfahrung, christliche Praxis, die Akzeptanz christlicher Rituale und christliches Wissen. Lag hier wie auch sonst in der Survey-Forschung der Fokus auf christlicher Religiosität, ${ }^{1}$ so wuchs

1 Bréchon (2009) vertritt sogar die These, dass die Art, wie Fragen (zur Religiosität) in einzelnen Umfrageprogrammen formuliert werden, konfessionell geprägt sei. So behauptet er, der European Value Study, in der überwiegend dichotome Items verwendet werden, sei, da hier nur zwischen „richtig“ und „falsch“ unterschieden wird, „katholisch“. Das ISSP hingegen, in welchem 
mit der Aufmerksamkeit für „,neue“ Religiositäts- und Spiritualitätsformen (vgl. Siegers 2012) und für die Zuwanderung von Muslimen, Juden und Angehörigen anderer Religionen das Interesse an Messinstrumenten, die im interreligiösen wie auch im internationalen Vergleich einsetzbar sind. Einen wichtigen Beitrag hat in dieser Hinsicht Stefan Huber (2003) geleistet. Er folgt zunächst Glock, teilt die Dimension des religiösen Verhaltens allerdings in private und öffentliche Formen und unterscheidet somit sechs Dimensionen der Religiosität, für die er zudem jeweils „Zentralität“" und „Inhalt“ unterscheidet (Huber 2007, S. 20). Am Beispiel transzendenter Vorstellungen bedeutet dies, dass ,zwischen der allgemeinen Intensität des Glaubens an eine Transzendenz und der Zustimmung zu verschiedenen Gottesbildern als inhaltliche Gestalten der Transzendenz unterschieden [wird]“ (Huber 2007, S. 20). Diese inhaltlichen Aussagen werden entweder so formuliert, dass sie unabhängig von einer Religion bedeutsam sind oder aber es werden den Befragten religionsspezifische Aussagen vorgelegt. Umfassend kam dieses Messinstrument im Religionsmonitor der Bertelsmann Stiftung zum Einsatz, einer international vergleichend angelegten Umfrage in insgesamt 21 Ländern aus Europa, Afrika, Asien, Süd- und Nordamerika (Bertelsmann Stiftung 2007), die innerhalb der Religionssoziologie für einiges Aufsehen sorgte. Den besonderen Anforderungen an die mehrdimensionale Messung von Religiosität wird auch in anderen innovativen Umfragen Rechnung getragen, so in der von Müller, Pickel und Pollack im Rahmen des Projekts „Kirche und Religion im erweiterten Europa“ durchgeführten internationalen Befragung oder in den von Diehl und Koenig verwendeten SCIP-Daten zu Neuzuwanderern.

Nach wie vor bleiben aber die in der zweiten Phase der Religionssoziologie entstandenen internationalen Surveys von großer Bedeutung, weil sie inzwischen anspruchsvolle Längsschnittuntersuchungen gestatten, wie sie in den Beiträgen von Roßteutscher und Wolf sowie in dem Beitrag von David Voas, Siobhan McAndrew und Ingrid Storm in diesem Sonderheft präsentiert werden. Letzterer zeichnet sich in methodischer Hinsicht zudem dadurch aus, dass er ein neues Instrument zur Untersuchung von Geschlechterunterschieden der Religiosität vorstellt. Mit seiner Hilfe zeigen sie, dass länderspezifische konfessionelle Prägungen und Säkularisierungsmuster sich auch darin niederschlagen, ob und wie schnell sich geschlechterspezifische Niveaus der Religiosität einander annähern.

Am oben erwähnten Religionsmonitor lässt sich, zweitens, aber auch die Bedeutung qualitativer Forschungsmethoden für die Religionssoziologie zeigen, die auf der Suche nach der von Luckmann vermuteten unsichtbaren Religion vielfach zum Einsatz kamen (vgl. Knoblauch 2003). In Ergänzung zur standardisierten Befragung wurden im Religionsmonitor 49 Leitfadeninterviews durchgeführt, die man dahingehend interpretiert hat, dass Menschen in Deutschland über eine durchaus hohe religiöse Kompetenz verfügen, dass ihre religiösen Vorstellungen jedoch nicht strikt einer Konfession folgen, sondern sich an vielen religiösen Traditionen orientieren (Nassehi 2007). In diesem Sonderheft zeigt sich die Stärke qualitativer Interviewmethoden, religiöse und säkulare Selbstverständnisse und deren lebensgeschichtliche Einbettung zu erfassen, insbesondere in den Beiträgen von Gärtner, Wohlrab-Sahr und Kaden, während die Beiträge von Kirsch

meist 4- oder 5-stufige Antwortskalen eingesetzt werden, sei differenzierter und damit „,protestantisch". 
sowie von Lüddeckens und Walthert die Relevanz ethnographischer Forschung für ein Verständnis gegenwärtiger Sozialformen des Religiösen dokumentieren.

Ein dritter Bereich der Methodenentwicklung hat seinen Ursprung im oben erläuterten Interesse an vergleichender Forschung zur Regulierung des religiösen Feldes. Entwickelte man zunächst anhand ausgewählter Indikatoren robuste Typologien für das Verhältnis von Staat und Kirche (Chaves und Cann 1992; Minkenberg 2003; Pollack 2003), so hat man inzwischen im Interesse globaler Vergleichsstudien auch quantifizierte Indizes gebildet. Grim und Finke (2006) beispielsweise versuchen mit Hilfe dreier sorgfältig operationalisierter Indizes den Grad staatlicher Bevorzugung einer bestimmten Religion, staatlicher Regulierung des religiösen Feldes und sozialer Regulierung des religiösen Feldes zu erfassen, während die Arbeiten von Fox (2008) eine Fülle von Detailinformationen zu verschiedensten Aspekten von Religionspolitik zusammentragen (zur kritischen Diskussion vgl. Traunmüller 2012). Ebenso wichtig für die makroquantitativ vergleichende Forschung ist die Operationalisierung des Grades religiöser Pluralisierung (vgl. Wolf 2012). In diesem Sonderheft stellt Richard Traunmüller eine hochdifferenzierte Messung religiöser Diversität vor, die religiöse Fraktionalisierung von Polarisierung unterscheidet und zudem deren Kongruenz mit soziostrukturellen Unterschieden abbildet. Dies gestattet ihm, in kritischer Weiterentwicklung der von Putnam angestoßenen Debatte um die Effekte von Diversität und unter Rückgriff auf internationale Daten nachzuweisen, dass verschiedene Modi religiöser Diversität sich höchst unterschiedlich auf soziales Vertrauen auswirken.

\section{Perspektiven der Religionssoziologie}

In unserer Einleitung zum vorliegenden Sonderheft „Religion und Gesellschaft“ der KZfSS haben wir den Versuch unternommen, die Situation der (deutschsprachigen) Religionssoziologie vor dem Hintergrund ihrer institutionellen und intellektuellen Entwicklung seit der Nachkriegszeit zu charakterisieren und einige aktuelle intellektuelle Trends zu identifizieren, die sich auch in den einzelnen Beiträgen des Sonderhefts niederschlagen. Zum Abschluss dieser Einleitung wollen wir ausblickartig einige Perspektiven für die zukünftige religionssoziologische Forschung benennen, die sich aus Desiderata der oben nachgezeichneten Debatten ableiten lassen.

Was den Bereich der Methodenentwicklung angeht, so wären angesichts der Spezialisierung quantitativer wie auch qualitativer Forschungsliteraturen Projekte wünschenswert, die beide methodische Ansätze fruchtbar miteinander verbinden. Solche Mixed-method-Ansätze könnten nicht nur dazu beitragen, den für die Soziologie insgesamt noch immer charakteristischen Methodendualismus aufzuweichen, sondern auch die dahinterstehenden theoretischen Positionen miteinander ins Gespräch zu bringen. Dabei sollten die international-vergleichenden Projekte der Erhebung von Mikro- und Makro-Daten gleichermaßen auch über den europäischen Kontext hinausgehend fortgesetzt werden. Dies nämlich käme der thematischen und regionalen Öffnung der Religionssoziologie entgegen, die angesichts ihrer langjährigen Zentrierung auf den europäischen Kontext dringend an der Zeit gewesen ist (vgl. ähnlich für die US-amerikanische Religionssoziologie Bender et al. 2012). Gerade die Erweiterung des regionalen Repertoires verspricht, 
präzisere Aussagen zur Reichweite (,scope conditions“) von Theoremen zu religiöser Assimilation, zur Organisierbarkeit von Religion, zur Dynamik globaler religiöser Bewegungen oder zu religionspolitischen Regimen treffen zu können. Gleichzeitig wäre zu wünschen, dass die entsprechenden Befunde der Religionssoziologie für angrenzende Teildisziplinen wie die Organisations-, die Migrations- oder die politische Soziologie systematisch fruchtbar gemacht würden.

Für die zukünftige Religionssoziologie wäre ferner gewinnbringend, die von der analytischen Soziologie und dem kritischem Realismus angestoßenen methodologischen Debatten um soziologisches Erklären weiterzuverarbeiten. Allerdings wird es wichtig sein, das mit der Geschichte der Teildisziplin konstitutiv verknüpfte Interesse an der Rekonstruktion allgemeiner Prozesse religiösen Wandels nicht aufzugeben. Die Herausforderung liegt vielmehr darin, regionalspezifische langfristige Trends der Religion in der Moderne aus dem historischen Zusammenspiel genereller kausaler Mechanismen zu erklären. Gerade durch eine solche Entwicklung würde auch die Säkularisierungstheorie vorangebracht werden, die, allen Ankündigungen des Kommens einer ,post-säkularen“ Konstellation zum Trotz, auch weiterhin in ihren verschiedenen Facetten die Fachdiskussion bestimmt. An Stelle der ursprünglichen narrativen Struktur der Modernisierungstheorie, die von Charles Taylor (2007) zu Recht für ihr epochales Stadienbewusstsein kritisiert wurde, träte dann allerdings eine mechanismenbasierte Rekonstruktion langfristiger Prozesse von Säkularisierung, von Privatisierung und De-Privatisierung von Differenzierung und Entdifferenzierung.

Die wohl schwierigste und anspruchsvollste Aufgabe zukünftiger religionssoziologischer Forschung liegt darin, die beiden derzeit zunehmend separat laufenden Traditionen des Religionsbegriffs wieder zusammenzuführen. Robert Bellahs (2011) evolutionstheoretischer Entwurf, der von einer Überlagerung (nicht Ablösung) ritueller Religion durch die achsenzeitlichen Transzendenzvisionen ausgeht, ist ein wichtiger Schritt in diese Richtung. Derartige Integrationsversuche sind deswegen von Bedeutung, weil sie helfen können, über die Fülle empirischer Detailfragen die theoretischen Leitprobleme, an denen sich in der Gründungsphase der Soziologie das Interesse an Religion ursprünglich entzündet hatte, insbesondere ihren Beitrag zu sozialer Ordnung und zu sozialem Wandel im Blick zu behalten.

Die in diesem Sonderheft versammelten Beiträge weisen aus unterschiedlichen disziplinären Perspektiven, mit unterschiedlichen theoretischen Orientierungen und auf Basis verschiedener empirischer Methoden und Daten auf all diese Desiderata hin. Würden sie der weiteren religionssoziologischen Debatte einige Impulse und Anstöße gegeben, so wäre die Absicht, die wir mit der Herausgabe des Sonderhefts verfolgt haben, erfüllt.

Danksagung: Wir danken der Fritz Thyssen Stiftung für die finanzielle und dem Mannheimer Zentrum für Europäische Sozialforschung für die organisatorische Unterstützung einer Autorentagung im Februar 2013, auf der die einzelnen Beiträge zu diesem Sonderheft intensiv diskutiert wurden. Unser Dank gebührt ferner Heike Antoni, Leonhard Link, Désirée Nießen und Maria Sinnemann für die Unterstützung während der Autorentagung sowie beim Korrekturlesen der einzelnen Beiträge und Marian Burchardt für konstruktive Kommentare zu dieser Einleitung. Wir danken den Gutachterinnen und Gutachtern für kritische Lektüre und hilfreiche Hinweise und vor allem Volker Dreier sowie dem Redaktionsteam der KZfSS für die umsichtige und geduldige Begleitung in den verschiedenen Entstehungsphasen dieses Sonderhefts. 


\section{Literatur}

Alexander, Jeffrey C. 2003. The meanings of social life: A cultural sociology. Oxford: Oxford University Press.

Asad, Talal. 1993. Genealogies of religion. Discipline and reasons of power in Christianity and Islam. Baltimore: John Hopkins University Press.

Beckford, James A. 2000. Start together and finish together: Shifts in the premises and paradigms underlying the scientific study of religion. Journal for the Scientific Study of Religion 4:481-495.

Bellah, Robert N. 2011. Religion in human evolution. From the palaeolithic to the axial Age. Cambridge: Belknap Press.

Bender, Courtney, Wendy Cadge, Peggy Levitt und David Smilde, Hrsg. 2012. Religion on the edge. De-centering and re-centering the sociology of religion. Oxford: Oxford University Press.

Berger, Peter L. 1973. Zur Dialektik von Religion und Gesellschaft. Elemente einer soziologischen Theorie. Frankfurt a. M.: Fischer.

Berger, Peter L. Hsrg. 1999. The desecularization of the World. Resurgent religion and world politics. Washington, D.C.: Ethics and Public Policy Center.

Berger, Peter L., Grace Davie und Effie Fokas. 2008. Religious America, secular Europe? A theme and variations. Aldershot: Ashgate.

Bergmann, Jörg, Alois Hahn und Thomas Luckmann, Hrsg. 1993. Religion und Kultur. Kölner Zeitschrift für Soziologie und Sozialpsychologie, Sonderheft 33. Opladen: Westdeutscher Verlag.

Bertelsmann Stiftung. 2007. Religions Monitor 2008. Gütersloh: Gütersloher Verlagshaus.

Beyer, Peter. 2006. Religions in global society. London: Routledge.

Boos-Nünning, Ursula. 1972. Dimensionen der Religiosität. Zur Operationalisierung und Messung religiöser Einstellungen. München: Kaiser.

Bourdieu, Pierre. 2000. Das religiöse Feld. Texte zur Ökonomie des Heilsgeschehens. Konstanz: Universitätsverlag Konstanz.

Brandt, Sigrid. 1993. Religiöses Handeln in moderner Welt. Talcott Parsons' Religionssoziologie im Rahmen seiner allgemeinen Handlungs- und Systemtheorie. Frankfurt a. M.: Suhrkamp.

Bréchon, Pierre. 2009. A breakthrough in comparative social research: The ISSP compared with the Eurobarometer, EVS and ESS surveys. In The International Social Survey Programme, Hrsg. Max Haller, Roger Jowell und Tom W. Smith, 28-44. New York: Routledge.

Breton, Raymond. 2012. Different gods. Integrating non-christian minorities into a primarily christian society. Montreal: McGill-Queen's University Press.

Brubaker, Rogers. 2012. Religion and nationalism: Four approaches. Nations and Nationalism 18:2-20.

Casanova, José. 1994. Public religions in the modern world. Chicago: Chicago University Press.

Casanova, José. 2006. Rethinking secularization: A global comparative perspective. The Hedgehog Review 8:7-22.

Chaves, Mark, und David E. Cann. 1992. Regulation, pluralism, and religious market structure. Explaining religion's vitality. Rationality and Society 4:272-290.

Daiber, Karl-Fritz, und Thomas Luckmann. Hrsg. 1983. Religion in den Gegenwartsströmungen der deutschen Soziologie. München: Kaiser.

Dobbelaere, Karel. 1981. Secularization. A multi-dimensional concept. London: Sage.

Ebertz, Michael N. 1987. Das Charisma des Gekreuzigten. Zur Soziologie der Jesusbewegung. Tübingen: Mohr-Siebeck.

Ebertz, Michael N. 1998. Erosion der Gnadenanstalt? Zum Wandel der Sozialgestalt von Kirche. Frankfurt a. M.: Knecht.

Eisenstadt, Shmuel N. 2000. Die Vielfalt der Moderne. Weilerswist: Velbrück Wissenschaft.

Fox, Jonathan. 2008. A world survey of religion and the state. Cambridge: Cambridge University Press. 
Gabriel, Karl, Christel Gärtner und Detlef Pollack. Hrsg. 2012. Umstrittene Säkularisierung: Soziologische und historische Analysen zur Differenzierung von Religion und Politik. Berlin: Berlin University Press.

Gabriel, Karl, Winfried Gebhardt and Michael Krüggeler. Hrsg. 1998. Institution - Organisation Bewegung. Sozialformen der Religion im Wandel. Opladen: Leske + Budrich.

Gill, Anthony. 2008. The political origins of religious liberty. Cambridge: Cambridge University Press.

Glock, Charles Y. 1962. On the study of religious commitment. Religious Education 57:98-110.

Goldschmidt, Dietrich, Franz Greiner und Helmut Schelsky, Hrsg. 1960. Soziologie der Kirchengemeinde. Stuttgart: Enke.

Goldschmidt, Dietrich, und Joachim Matthes. Hrsg. 1962. Probleme der Religionssoziologie. Kölner Zeitschrift für Soziologie und Sozialpsychologie, Sonderheft 6. Köln: Westdeutscher Verlag.

Gorski, Philip S. 2000. Historicizing the secularization debate. American Sociological Review 65:138-167.

Gorski, Philip S. 2003. The disciplinary revolution. Calvinism and the rise of the state in early modern Europe. Chicago: University of Chicago Press.

Gorski, Philip S. 2009. Social ,mechanisms` and comparative-historical sociology: A critical realist proposal. In Frontiers of Sociology, Hrsg. Peter Hedström und Björn Wittroc, 147-194. Leiden: Brill.

Gorski, Philip S., und Ateş Altinordu. 2008. After secularization? Annual Review of Sociology 34:55-85.

Graf, Friedrich Wilhelm. 2004. Die Wiederkehr der Götter. Religion in der modernen Kultur. München: Beck.

Grim, Brian, und Roger Finke. 2006. International religion indexes: Government regulation, government favoritism and social regulation of religion. Interdisciplinary Journal of Research and Religion 2:1-40.

Grim, Brian J., und Roger Finke. 2011. The price of freedom denied. Religious persecution and conflict in the twenty-first century. Cambridge: Cambridge University Press.

Habermas, Jürgen. 2001. Glauben und Wissen. Rede anlässlich der Verleihung des Friedenspreises des Deutschen Buchhandels. Frankfurt a. M.: Suhrkamp.

Hahn, Alois. 1982. Zur Soziologie der Beichte und anderer Formen institutionalisierter Bekenntnisse: Selbstthematisierung und Zivilisationsprozess. Kölner Zeitschrift für Soziologie und Sozialpsychologie 34:407-434.

Hahn, Alois. 1986. Differenzierung, Zivilisationsprozess, Religion. Aspekte einer Theorie der Moderne. In Kultur und Gesellschaft, Kölner Zeitschrift für Soziologie und Sozialpsychologie, Sonderheft 27, Hrsg. Friedhelm Neidhardt, M. Rainer Lepsius und Johannes Weiß, 214-231. Opladen: Westdeutscher Verlag.

Hedström, Peter. 2005. Dissecting the social. On the principles of analytical sociology. Cambridge: Cambridge University Press.

Heelas, Paul, und Linda Woodhead. 2005. The spiritual revolution. Why religion is giving way to spirituality. Oxford: Blackwell.

Hennig, Anja. 2012. Moralpolitik und Religion. Bedingungen politisch-religiöser Kooperation in Polen, Italien und Spanien. Würzburg: Ergon-Verlag.

Hero, Markus. 2010. Die neuen Formen des religiösen Lebens. Eine institutionentheoretische Analyse neuer Religiosität. Würzburg: Ergon.

Hervieu-Léger, Daniele. 2004. Pilger und Konvertiten. Religion in Bewegung. Würzburg: Ergon-Verlag.

Höllinger, Franz. 2007. Religiöse Kultur in Brasilien. Zwischen traditionellem Volksglauben und modernen Erweckungsbewegungen. Frankfurt a. M.: Campus. 
Huber, Stefan. 2003. Zentralität und Inhalt. Ein neues multidimensionales Messmodell der Religiosität. Opladen: Leske + Budrich.

Huber, Stefan. 2007. Analysen zur religiösen Praxis. Ein Blick in die Schweiz. In ReligionsMonitor 2008, Hrsg. Bertelsmann Stiftung, 158-166. Gütersloh: Gütersloher Verlagshaus.

Inglehart, Ronald, und Pippa Norris. 2003. Rising tide. Gender equality and cultural change around the world. Cambridge: Cambridge University Press.

Joas, Hans. 2004. Braucht der Mensch Religion? Freiburg: Herder.

Joas, Hans. 2011. Die Sakralität der Person. Eine neue Genealogie der Menschenrechte. Berlin: Suhrkamp.

Kecskes, Robert, und Christof Wolf. 1993. Christliche Religiosität: Konzepte, Indikatoren, Meßinstrumente. Kölner Zeitschrift für Soziologie und Sozialpsychologie 45:270-287.

Kecskes, Robert, und Christof Wolf. 1995. Christliche Religiosität: Dimensionen, Meßinstrumente, Ergebnisse. Kölner Zeitschrift für Soziologie und Sozialpsychologie 47:494-515.

Kepel, Gilles. 1991. La revanche de Dieu: Chrétiens, Juifs, Musulmans à la reconquête du monde. Paris: Seuil.

Kippenberg, Hans G. 2008. Gewalt als Gottesdienst. Religionskriege im Zeitalter der Globalisierung. München: Beck.

Knoblauch, Hubert. 1989. Das Unsichtbare Zeitalter. ,New Age‘, privatisierte Religion und kultisches Milieu. Kölner Zeitschrift für Soziologie und Sozialpsychologie 41:503-525.

Knoblauch, Hubert. 2003. Qualitative Religionsforschung: Religionsethnographie in der eigenen Gesellschaft. Paderborn: Schöningh.

Knöbl, Wolfgang. 2007. Die Kontingenz der Moderne. Wege in Europa, Asien und Amerika. Frankfurt a. M.: Campus.

Koenig, Matthias. 2007. Europäisierung von Religionspolitik. Zur institutionellen Umwelt der Anerkennungskämpfe muslimischer Migranten. Soziale Welt - Sonderheft ,Islam in Europa “ 17:345-367.

Koenig, Matthias. 2011. Jenseits der Säkularisierungstheorie? Zur Auseinandersetzung mit Charles Taylor. Kölner Zeitschrift für Soziologie und Sozialpsychologie 63:649-673.

Krech, Volkhard. 1998. Georg Simmels Religionstheorie. Tübingen: Mohr.

Krech, Volkhard, und Hartmut Tyrell. Hrsg. 1995. Religionssoziologie um 1900. Würzburg: Ergon-Verlag.

Kuru, Ahmet T. 2009. Secularism and state policies toward religion. The United States, France, and Turkey. Cambridge: Cambridge University Press.

Luckmann, Thomas. 1960. Neuere Schriften zur Religionssoziologie. Kölner Zeitschrift für Soziologie und Sozialpsychologie 12:315-326.

Luckmann, Thomas. 1991. Die unsichtbare Religion. 1967. Frankfurt a. M.: Suhrkamp.

Luhmann, Niklas. 1972. Die Organisierbarkeit von Religion und Kirchen. In Religion im Umbruch. Soziologische Beiträge zur Situation von Religion und Kirche in der gegenwärtigen Gesellschaft, Hrsg. Jakobus Wössner, 245-285. Stuttgart: Enke.

Luhmann, Niklas. 1977. Funktion der Religion. Frankfurt a. M.: Suhrkamp.

Luhmann, Niklas. 2000. Die Religion der Gesellschaft. Frankfurt a. M.: Suhrkamp.

Lynch, Gordon. 2012. The sacred in the modern world. A cultural sociological approach. Oxford: Oxford University Press.

Manow, Philip. 2008. Religion und Sozialstaat. Die konfessionellen Grundlagen europäischer Wohlfahrtsstaatsregime. Frankfurt a. M.: Campus.

Martin, David. 1990. Tongues of fire. The explosion of protestantism in Latin America. Oxford: Blackwell.

Marty, Martin E., und R. Scott Appleby. Hrsg. 1991. Fundamentalisms observed. Chicago: University of Chicago Press. 
Matthes, Joachim. 1962. Bemerkungen zur Säkularisierungsthese in der neueren Religionssoziologie. In Probleme der Religionssoziologie, Kölner Zeitschrift für Soziologie und Sozialpsychologie, Sonderheft 6, Hrsg. Dietrich Goldschmidt und Joachim Matthes, 65-77. Köln: Westdeutscher Verlag.

Matthes, Joachim. 1993. Was ist anders an anderen Religionen? Anmerkungen zur zentristischen Organisation des religionssoziologischen Denkens. In Religion und Kultur. Kölner Zeitschrift für Soziologie und Sozialpsychologie, Sonderheft 33, Hrsg. Jörg R. Bergmann, Alois Hahn und Thomas Luckmann, 16-30. Opladen: Westdeutscher Verlag.

McLeod, Hugh. 2000. Secularisation in Western Europe, 1848 - 1914. Houndmills, Basingstoke: Macmillan Press.

Minkenberg, Michael. 2003. Staat und Kirche in westlichen Demokratien. In Politik und Religion. Politische Vierteljahresschrift, Sonderheft 33, Hrsg. Michael Minkenberg und Ulrich Wilhems, 115-138. Baden-Baden: Nomos.

Möbius, Stephan. 2013. Das Collège de Sociologie und die Religionssoziologie der DurkheimSchule. In Émile Durkheim. Soziologie - Ethnologie - Philosophie, Hrsg. Tanja Bogusz und Heike Delitz, 473-501. Frankfurt a. M.: Campus.

Nassehi, Armin. 2007. Erstaunliche religiöse Kompetenz. Qualitative Ergebnisse des Religionsmonitors. In ReligionsMonitor 2008, Hrsg. Bertelsmann Stiftung, 113-134. Gütersloh: Gütersloher Verlagshaus.

Norris, Pippa, und Ronald Inglehart. 2004. Sacred and secular. Religion and politics worldwide. Cambridge: Cambridge University Press.

Pickel, Gert. 2010. Säkularisierung, Individualisierung oder Marktmodell? Religiosität und ihre Erklärungsfaktoren im europäischen Vergleich. Kölner Zeitschrift für Soziologie und Sozialpsychologie 62:219-246.

Platvoet, Jan G., und Arie L. Molendijk. Hrsg. 1999. The pragmatics of defining religion. Contexts, concepts and contests. Leiden: Brill.

Pollack, Detlef. 1995. Was ist Religion? Probleme der Definition. Zeitschrift für Religionswissenschaft 3:163-190.

Pollack, Detlef. 2003. Säkularisierung - ein moderner Mythos? Studien zum religiösen Wandel in Deutschland. Tübingen: Siebeck.

Pollack, Detlef. 2009. Rückkehr des Religiösen? Tübingen: Mohr Siebeck.

Pollack, Detlef, Ingrid Tucci und Hans-Georg Ziebertz. Hrsg. 2012. Religiöser Pluralismus im Fokus quantitativer Religionsforschung. Wiesbaden: Springer VS.

Putnam, Robert, und David E. Campbell. 2010. American grace. How religion divides and Unites Us. New York: Simon \& Schuster.

Reckwitz, Andreas. 2001. Die Transformation der Kulturtheorie. Weilerswist: Velbrück Wissenschaft.

Reigrotzki, Erich. 1956. Soziale Verflechtungen in der Bundesrepublik. Elemente der sozialen Teilnahme in Kirche, Politik, Organisation und Freizeit. Tübingen: Mohr.

Riesebrodt, Martin. 1990. Fundamentalismus als patriarchalische Protestbewegung. Amerikanische Protestanten (1910-28) und iranische Schiiten (1961-79) im Vergleich. Tübingen: Mohr.

Riesebrodt, Martin. 2000. Rückkehr der Religionen? Zwischen Fundamentalismus und „Kampf der Kulturen “. München: Beck.

Riesebrodt, Martin. 2007. Cultus und Heilversprechen. Eine Theorie der Religionen. München: Beck.

Schluchter, Wolfgang. 1988. Religion und Lebensführung. Studien zu Max Webers Religions- und Herrschaftssoziologie. Frankfurt a. M.: Suhrkamp.

Siegers, Pascal. 2012. Alternative Spiritualitäten. Neue Formen des Glaubens in Europa: Eine empirische Analyse. Frankfurt a. M.: Campus.

Smith, Christian. Hrsg. 2003. The secular revolution: Power, interest, and conflict in the secularization of American public life. Berkeley: University of California Press. 
Smith, Christian. 2010. What is a person? Rethinking humanity, social life, and the moral good from the person up. Chicago: University of Chicago Press.

Spohn, Willfried. 2008. Politik und Religion in einer sich globalisierenden Welt. Wiesbaden: VS Verlag für Sozialwissenschaften.

Stark, Rodney. 1999. Micro-foundations of religion - A revised theory. Sociological Theory 17:264-89.

Stark, Rodney, und Laurence R. Iannaccone. 1994. A supply-side reinterpretation of the „Secularization" of Europe. Journal for the Scientific Study of Religion 33:230-252.

Taylor, Charles. 2007. A secular age. Cambridge: The Belknap Press of Harvard University Press.

Tezcan, Levent. 2003. Das Islamische in den Studien zu Muslimen in Deutschland. Zeitschrift für Soziologie 32:237-261.

Traunmüller, Richard. 2012. Zur Messung von Staat-Kirche-Beziehungen. Eine vergleichende Analyse neuerer Indizes. Zeitschrift für Vergleichende Politikwissenschaft 6:207-231.

Tschannen, Olivier. 1992. Les théories de la sécularisation. Genève: Librairie DROZ.

Tyrell, Hartmann. 1993. Potenz und Depotenzierung der Religion - Religion und Rationalisierung bei Max Weber. Saeculum 44:300-347.

Tyrell, Hartmann Volkhard Krech und Hubert Knoblauch. Hrsg. 1998. Religion als Kommunikation. Würzburg: Ergon Verlag.

van der Veer, Peter. 2001. Imperial encounters. Religion and modernity in India and Britain. Princeton: Princeton University Press.

Vidich, Arthur J., und Stanford M. Lyman. 1985. American sociology: Worldly rejections of religion and their directions. New Haven: Yale University Press.

Voas, David. 2009. The rise and fall of Fuzzy Fidelity in Europe. European Sociological Review 25:155-168.

Willaime, Jean-Paul. 1999. French-language sociology of religion in Europe since the Second World War. Revue Suisse de Sociologie 25:343-371.

Willems, Ulrich, Detlef Pollack, Helene Basu, Thomas Gutmann and Ulrike Spohn. Hrsg. 2013. Moderne und Religion. Kontroversen um Modernität und Säkularisierung. Bielefeld: transcript.

Wohlrab-Sahr, Monika. 2000. „Luckmann 1960“ und die Folgen. Neuere Entwicklungen in der deutsch-sprachigen Religionssoziologie. Soziologie 25:36-60.

Wohlrab-Sahr, Monika, und Marian Burchardt. 2012. Multiple secularities: Toward a cultural sociology of secular modernities. Comparative Sociology 11:1-35.

Wohlrab-Sahr, Monika, Uta Karstein und Thomas Schmidt-Lux. 2009. Forcierte Säkularität: religiöser Wandel und Generationendynamik im Osten Deutschlands. Frankfurt a. M.: Campus.

Wolf, Christof. 2005. Measuring religious affiliation and religiosity in Europe. In Methodological aspects in cross-national research, Hrsg. Jürgen H. P. Hoffmeyer-Zlotnik und Janet Harkness, 279-294. Mannheim: ZUMA Mannheim.

Wolf, Christof. 2012. Konzepte zur Messung religiöser Pluralität. In Religiöser Pluralismus im Fokus quantitativer Religionsforschung, Hrsg. Detlef Pollack, Ingrid Tucci und Hans-Georg Ziebertz, 17-38. Wiesbaden: Springer VS.

Zuckermann, Phil. 2009. Society without God. What the least religious nations can tell us about contentment. New York: New York University Press.

Matthias Koenig, 1971, Prof. Dr. phil., Institut für Soziologie, Georg-August-Universität Göttingen. Forschungsgebiete: Religionssoziologie, historisch-komparative Soziologie, soziologische Theorie. Veröffentlichungen u. a.: Religionskontroversen in Frankreich und Deutschland. Hamburg 2008 (hrsg. mit J.-P. Willaime); International migration and the governance of religious diversity. Montreal, Kanada 2009 (hrsg. mit P. Bramadat); Jenseits des Säkularisierungsparadigmas? Eine Auseinandersetzung mit Charles Taylor. Kölner Zeitschrift für Soziologie und Sozialpsychologie 63, 2012. 
Christof Wolf, 1963, Prof. Dr., GESIS - Leibniz-Institut für Sozialwissenschaften und Universität Mannheim. Forschungsgebiete: Sozialstruktur moderner Gesellschaften, Religionssoziologie, quantitative Methoden und Datenanalyse. Veröffentlichungen: Handbuch der sozialwissenschaftlichen Datenanalyse. Wiesbaden 2010 (mit H. Best); Konzepte zur Messung religiöser Pluralität. In: D. Pollack et al. (Hrsg.): Religiöser Pluralismus im Fokus quantitativer Religionsforschung, Wiesbaden 2012; How Secularized is Germany? Cohort and Comparative Perspectives. Social Compass $55,2008$. 